\title{
INVESTIGACIÓN EN AULA EN EDUCACIÓN DE ADULTOS: EL PROYECTO NUESTRA ESCUELA PREGUNTA SU OPINIÓN-NEPSO
}

\author{
Guillermo Willianson* \\ Issa Torres B.** \\ Natalia Durán $\mathrm{M}^{* * *}$
}

RESUMEN: El artículo reflexiona sobre aprendizajes de la aplicación de una metodología de investigación participativa en aula, basada en encuestas de opinión (Nuestra Escuela Pregunta Su Opinión-NEPSO). Uno de los niveles educativos en que se desarrolla es el de la Educación de Adultos. Desde el año 2005 el Centro Integrado de Educación de Adultos Lefxarv de la Región de La Araucanía en Chile lo impulsa con un proyecto educativo intercultural. Esta innovación pedagógica ha ido abarcando el conjunto de los diversos niveles en que este CEIA estructura su organización curricular: metodología de investigación en aula a través de encuestas de opinión. El artículo presenta su proceso pedagógico y resultados referidos a logros de aprendizajes, integración curricular, diversidad y trabajo docente, reflexiona sobre ellos y discute una evaluación a partir de un estudio de impactos de la metodología en los aprendizajes y de otra información analítica complementaria recogida desde los procesos de autoformación docente y de sistematización.

Palabras claves: Investigación. Educación de Adultos. Currículo.

\footnotetext{
* Magíster en Educación por la P. Universidad Católica de Chile y Doctor en Educación por la Universidad Estadual de Campinas, Campinas-SP, Brasil. Profesor Asociado y Director del Departamento de Educación de la Universidad de La Frontera, Temuco, Chile, donde ejerce desde 1994. Dirige la Línea de Educación de Adultos y el Proyecto Nuestra Escuela Pregunta Su Opinión-NEPSO de la Universidad de La Frontera. E-mail: gwilliam@ufro.cl

** Profesora de Estado en Castellano y Comunicación y Licenciada en Educación de la Universidad de La Frontera. Actualmente es profesora del Sector de Lenguaje y Comunicación en el Centro Integrado de Educación de Adultos Lefxarv de Nueva Imperial, Región de La Araucanía. E-mail: issatorres2006@hotmail.com

*** Profesora de Estado en Castellano y Comunicación y Licenciada en Educación de la Universidad de La Frontera. Actualmente es profesora del Sector de Lenguaje y Comunicación en el Colegio Laico Concepción de Angol, Región de La Araucanía. E-mail: dathy_84@hotmail.com
} 


\section{INVESTIGATION INTO ADULT EDUCATION TEACHING: \\ THE PROJECT ‘OUR SCHOOL ASKS YOUR OPINIÓN'-NEPSO}

ABSTRACT: The article reflects on learning outcomes arising from the application of a participative, investigative methodology in the classroom, based on the questionnaire (Our School Asks Your Opinion - NEPSO). One of the educational levels in which it has been evolving, is that of Adult Education. Since 2005, the Integrated Adult Education Centre, Lefxarv of the Araucania Region, Chile, has helped to develop it with their Inter-Cultural Education Project. This pedagogical innovation has managed to include the diverse set of levels that the CEIA uses to structures its curriculum: a classroom investigation methodology dependant on the use of public opinion surveys. This article presents the pedagogical process and the results, referring to the learning achievements, curricular integration, diversity and educational work, reflecting on these and discussing an evaluation which commences from an impact study of the methodology and the learning achievements and of other complementary analytical information, identified from the outset of the self developmental educational processes and their systematisation. Keywords: Investigation. Adult Education. Curriculum.

\section{Antecedentes}

Desde el año 2005 en la Universidad de La Frontera (UFRO), Chile, se desarrolla en el ámbito educacional de adultos una experiencia de innovación pedagógica centrada en proyectos de aula, integración curricular y cooperación docente: el Proyecto Nuestra Escuela Pregunta Su Opinión (NEPSO) $^{1}$ (WILLIAMSON, 2008). El objetivo de este trabajo es promover y difundir la utilización de la metodología de encuesta de opinión como una alternativa efectiva de innovación pedagógica en el trabajo de aula en escuelas públicas de enseñanza regular básica y media y en cursos de Educación de Jóvenes y Adultos (EDJA) orientada al mejoramiento de los aprendizajes.

Los tres ejes centrales que constituyen la metodología son: la integración curricular; la metodología de proyectos de investigación; la cooperación docente. Estos tres aspectos se plantean como una unidad en que el conocimiento que constituye el producto del proceso de aprendizaje se produce cooperativamente por la acción pedagógica de los docentes y el protagonismo de los que aprenden. Además, el conocimiento que se produce en el proceso de investigación debe ser coherente entre la realidad como una unidad holística y sistémica, la cognición que organiza relaciones y contenidos culturales en saberes complejos y los sentidos y significados culturales de los individuos y sus comunidades. 
La metodología propuesta para el desarrollo de proyectos de encuestas de opinión propicia aprendizajes significativos que atienden a las orientaciones curriculares actuales para la educación básica y media de adultos. Este enfoque constituye una experiencia de práctica escolar que concreta los principios de contextualización de contenidos, integración de disciplinas, valoración de la iniciativa y autonomía de los jóvenes, ciudadanía y participación, creando posibilidades de innovación del trabajo pedagógico y de los procesos y estilos de aprendizaje.

El proyecto cuenta con el apoyo de la empresa TimeIbope ${ }^{2}$ de Chile, el Instituto Brasileño de Opinión Pública y Estadística (IBOPE) ${ }^{3}$, a través del Instituto Paulo Montenegro, y la Organización No Gubernamental (ONG) Ação Educativa de Brasil ${ }^{5}$. El Polo Chile es parte de una Red Iberoamericana de Polos ${ }^{6}$ conectados a través de la Web, que realiza actividades y encuentros pedagógicos periódicos. De esta red forman parte Brasil (São Paulo, Rio de Janeiro, Pernambuco, Paraná, Minas Gerais y Rio Grande do Sul), México, Argentina, Chile y Colombia, en América Latina, y Portugal, en Europa. ${ }^{7}$ En los encuentros se implementan anualmente varios cientos de proyectos sobre temas diversos y que abarcan distintos cursos, temas y sectores disciplinarios, en la enseñanza básica, media y de adultos (INSTITUTO PAULO MONTENEGRO, 2007).

El siguiente cuadro muestra los indicadores cuantitativos del desarrollo del proyecto NEPSO desde el año 2005.

\section{CUADRO 1}

NÚMERO DE ESTABLECIMIENTOS EDUCATIVOS POR AÑO - POLO CHILE

\begin{tabular}{c|c|c|c|c|c|c} 
Nivel & $\mathbf{2 0 0 5}$ & $\mathbf{2 0 0 6}$ & $\mathbf{2 0 0 7}$ & $\mathbf{2 0 0 8}$ & $\mathbf{2 0 0 9}$ & T0TAL \\
\hline Básica & & & & 1 & 5 & $\mathbf{6}$ \\
\hline Media & 1 & 1 & 5 & 4 & 1 & $\mathbf{1 5}$ \\
\hline Adulto & 2 & 1 & 1 & 1 & 1 & $\mathbf{6}$ \\
\hline Superior & & & 1 & $\mathbf{6}$ & $\mathbf{1 2}$ & $\mathbf{3 1}$
\end{tabular}

Uno de los niveles en que se desarrolla el proyecto es el de la educación de adultos (EDA). Desde el año 2005 el Centro Integrado de Educación de Adultos (CEIA) Lefxarv ${ }^{8}$ lo impulsa con un proyecto educativo intercultural. Esta innovación pedagógica progresivamente ha ido abarcando el conjunto de los diversos niveles en que el CEIA estructura 
su organización curricular: metodología de investigación de encuestas de opinión en aula. Este artículo presenta su proceso pedagógico y resultados referidos al aprendizaje, reflexiona sobre él y discute una evaluación de procesos y resultados a partir de un estudio evaluativo de impactos de la metodología en los aprendizajes y de otra información evaluativa complementaria recogida desde los procesos de autoformación docente y de sistematización.

\section{EI CEIA Lefxarv ${ }^{9}$}

La Asociación Comunal Mapuche de Nueva Imperial (Región de La Araucanía, Comuna de Nueva Imperial), territorio histórico del pueblo mapuche, nace como una necesidad de diferentes organizaciones territoriales de afrontar el nuevo escenario que les presenta el Estado de Chile una vez retornada la Democracia (Ley Indígena 19.253 del 5 de Octubre de 1993). El objetivo de los mapuche era crear una figura legal que les permitiese interactuar con la administración del Estado y acceder a los diferentes instrumentos de promoción y fomento que el Estado ofrecía. La Asociación existe desde 1995, en el contexto de la Ley indígena, reuniendo a 9 organizaciones de nivel comunitario, participando de las iniciativas de coordinación de las diversas identidades territoriales. El CEIA Lefxarv fue reconocido por el Ministerio de Educación por resolución exenta $\mathrm{N}^{\circ}$ 0240, en 3 de marzo de 1999, a la fecha este establecimiento ha cumplido 10 años brindando un servicio de Educación Intercultural Bilingüe en la región. Su misión es "potenciar el desarrollo de la identidad, capital social y cultural disponible en la población joven y adulta que atiende en un ambiente de respeto, cooperación e interrelación del saber de la sociedad mapuche y la sociedad global". Cuenta con un sector de Formación General Básica con Oficios: en Tercer Nivel Básico: Gürrekan zugu (Artesano Textil); en el Segundo Nivel Básico: Iyael zugu (Manipulador de Alimentos) y en el Tercer Nivel Básico: Lawen zugu (Remedios Naturales).

El CEIA Lefxarv desarrolla un Proyecto Educativo Institucional, en el que la interculturalidad es un objetivo transversal y se caracteriza por contar con un equipo de profesores hablantes de la Lengua Mapuche-Mapucezugun. El Proyecto Educativo exige enseñar la lengua mapuche a todos los estudiantes que han asistido y asisten a los 
distintos niveles de Enseñanza Básica y Media. Desde el año 2004, cuenta con un Plan y programa propio de Lengua y Cultura Mapuche reconocido por el Ministerio de Educación. Es un espacio para preservar y revitalizar la lengua Mapuche-Mapucezugun y promover la artesanía y las actividades comunitarias mapuche: We xipantu, Palin, Rukan. Participa con los estudiantes en la rogativa más importante del Ser Mapuche, el Gijatun.

\section{Los proyectos de investigación en aula en el CEIA}

Los temas generales y problemas específicos de los proyectos de investigación que se han desarrollado en el CEIA son definidos por los propios estudiantes, como lo exige la metodología, en el marco de los sectores de aprendizaje definidos por los docentes. Cada proyecto se realiza en general en unos seis o siete meses y no forman parte de actividades especiales o extra-aula, sino de acciones integradas directamente a las prácticas docentes y aprendizajes de los estudiantes según el currículo y aulas habituales.

Los temas tratados por año en el CEIA se muestran en el siguiente cuadro:

\section{CUADRO 2}

\begin{tabular}{|c|c|}
\hline AÑ̃ & PROYECTOS \\
\hline 2005 & (Año de perfeccionamiento de los docentes) \\
\hline 2006 & Distribución de ingresos familiares de la ciudad de Imperial \\
\hline 2007 & $\begin{array}{l}\text { Adolescentes de Imperial: ¿realmente informados sobre sus } \\
\text { derechos sexuales y reproductivos? }\end{array}$ \\
\hline 2008 & $\begin{array}{l}\text { 1. Animales callejeros: ¿Problema Social o Indiferencia? } \\
\text { 2. Uso y percepción de las plantas medicinales en la comuna de Nueva Imperial }\end{array}$ \\
\hline 2009 & $\begin{array}{l}\text { 1. La vida en pareja, preferencia de la convivencia o el matrimonio } \\
\text { 2. Calidad de la atención en salud pública } \\
\text { 3. Drogadicción: estrategias de preservación que usan las familias para } \\
\text { prevenir el uso de drogas }\end{array}$ \\
\hline
\end{tabular}

Los resultados de estos proyectos de aula, que integran varios sectores o disciplinas (asignaturas) en torno a una que hace de eje, son evaluados y calificados, formando parte de los promedios anuales de promoción. Además, los resultados son presentados a la comunidad escolar y local en reuniones especiales y en los encuentros nacionales (2007 y 2009) o internacionales (2008) organizados cada año por la UFRO (WILLIAMSON, 2008). 


\section{Resultados de un estudio sobre los aprendizajes logrados por el desarrollo de la metodología}

Las sistematizaciones y estudios realizados muestran que la metodología genera cambios y mejoramientos en diversos niveles, sectores y resultados de competencias de aprendizaje cognitivos, sociales e instrumentales que permiten fortalecer la autoestima, sociabilidad, ciudadanía y capacidad comunicativa de los estudiantes. Sin embargo señalan también carencias y desafíos en cuanto a la evaluación y a la falta de indicadores e instrumentos más precisos, permanentes y diversos. La observación y medición más detallada de logros de aprendizajes y de cambios personales permitirían mejorar o complementar la metodología (BARRÍA, PAMELA, 2008; DURÁN MONDACA, TORRES BRAVO, 2008; WILLIAMSON, 2009).

El año 2007 se realizó una investigación - una tesis para obtener el grado de Licenciatura en Ecuación - respecto del desarrollo y resultados de la metodología del CEIA que implicó el primer registro sistemático de los pasos en que este proyecto se desarrolló en aula durante un año y que, junto a ello, observó y evaluó resultados de aprendizaje (DURÁN MONDACA, TORRES BRAVO, 2008).

A continuación se presentan los principales resultados de este estudio que arrojan información inicial para un proceso más complejo de evaluación de la metodología y el proyecto.

\subsection{La metodología}

La tesis realizada, durante el año 2007, con una metodología cualitativa de investigación acción participativa (WILLIAMSON, 2002) recogió la experiencia que se obtuvo mediante la práctica del NEPSO en el Centro de Educación Intercultural de Adultos Lefxarv, Imperial, Región de la Araucanía. Se sustentó en la observación sistemática - con una pauta diseñada por las investigadoras para verificar cambios como logros de aprendizaje y participación directa de las investigadoras, en las conversaciones y entrevistas a informantes claves y en la revisión de registros documentales.

Los datos básicos de contexto de la investigación son los siguientes: 


\section{CUADRO DE INFORMACIÓN BÁSICA Y DE CONTEXTO DEL PROYECTO}

\begin{tabular}{c|c} 
Establecimiento educacional & CEIA Lefxarv \\
\hline Directora & Irene Huentemilla \\
\hline Profesores(as) participantes & $\begin{array}{c}\text { Irene Huentemilla (Biología) } \\
\text { Gloria Marivil (Estudios Sociales) } \\
\text { Daniel Toro (Matemáticas) }\end{array}$ \\
\hline Alumnas tesistas investigadoras & Natalia Durán \\
Issa Torres
\end{tabular}

A través de una pauta elaborada por las propias investigadoras se definieron indicadores de observación de aprendizajes (cambios) en relación a los sectores Lenguaje, Matemática, Ciencias, Tecnología y a aspectos motivacionales.

\subsection{La situación inicial del curso observado}

Las características socio-económicas corresponden a los alumnos y alumnas del $2^{\circ}$ ciclo del Centro de Educación Intercultural Lefxarv, año 2007. El curso estaba compuesto por 20 adultos de distintas edades, entre 18 y 60 años, 5 de ellos participantes de una primera experiencia NEPSO durante el año 2006. Un 50\% provenía de comunidades mapuche, los restantes no pertenecían a esta etnia y vivían en la ciudad de Nueva Imperial. Sus ocupaciones eran diversas, un 65\% desarrollaba actividades remuneradas fuera del hogar, mientras que un $20 \%$ se encontraba sin trabajo. Un 15\% desarrollaba labores domésticas no remuneradas y el 5\% restante se dedicaba sólo a estudiar. Desde el punto de vista económico la mayoría pertenecía a familias con bajos ingresos.

A partir del diagnóstico inicial se observaron, al comenzar a aplicarse la metodología NEPSO, una serie de dificultades de aprendizaje en los distintos sectores. En los sectores de Comunicación y Estudios Sociales los alumnos mostraron bajo nivel de comprensión lectora, dificultades para producir textos con coherencia y cohesión, inseguridad en las actividades de expresión verbal o escrita formal. En el ámbito de las Matemáticas y Ciencias (Naturales) presentaron dificultades para aplicar operatoria básica frente a la resolución y comprensión de problemas de la vida diaria y entorno. 


\subsection{La sistematización del proceso}

El proyecto en lo metodológico tiene una serie de etapas que están definidas en un manual metodológico NEPSO (MONTENEGRO, FABIO \& MASAGAO, VERA, 2002) y en una serie de materiales didácticos que constituyen un conjunto de textos orientadores para los docentes. $)^{10}$

La primera etapa del proceso de investigación consiste en la definición democrática, por los estudiantes, de un tema y, luego, un problema específico dentro de él que será el que se investigue con la metodología de encuesta de opinión. En una de las reuniones de comunidad autogestionada de aprendizaje de los docentes, la Directora del CEIA y profesora de Biología Irene Huentemilla describe de la siguiente manera esta primera actividad en la que se reflejan los principios metodológicos del proyecto, la dinámica pedagógica y curricular y el papel de los docentes y estudiantes en él:

El tema lo eligieron los alumnos con metodologías que definieron próximas a las de educación popular, fue una metodología en que dibujaron sus manos y ahí escribieron sus problemas, en los dedos del dibujo anotaban una característica de cada uno y en la palma la pregunta o tema que les interesaba investigar. Como son madres y mujeres jóvenes les interesó la situación juvenil: droga, alcohol, embarazo adolescente... No me gusta mucho lo de la votación, pues el año pasado yo quería un tema, pero ellos eligieron otro, este año pasó lo mismo... Pero, en fin, así es la metodología, democrática. La profesora de historia no sabía como articularlo en el primer semestre, pues no correspondía a su programa; le expliqué que la idea del NEPSO es que todo se articule y se integre; entonces ella va a ver lo del adolescente desde la perspectiva cultural en diversos países y culturas, entre ellas lo de la cultura mapuche y el embarazo (WILLIAMSON, 2008, p. 21).

Las etapas se organizaron en los siguientes contenidos y secuencia: 


\section{ETAPAS Y ACTIVIDADES DEL PROYECTO}

\begin{tabular}{|c|c|c|c|}
\hline ETAPA METODOLÓGICA & MES & díA & DESCRIPCIÓN DE LA ACTIVIDAD \\
\hline \multirow[t]{4}{*}{ PLANIFICACIÓN } & \multirow[t]{2}{*}{ Abril 2007} & 5 & Presentación global del proyecto NEPSO. \\
\hline & & 12 & $\begin{array}{l}\text { Experiencia NEPSO - Lefxavu } 2006 \text { contada por el grupo de } \\
\text { alumnos que participó ese año. } \\
\text { Discusión. }\end{array}$ \\
\hline & & 19 & $\begin{array}{l}\text { Encuesta evaluación inicial. } \\
\text { Resumen de los recursos: (23 alumnos, computadores, internet, libros). }\end{array}$ \\
\hline & & 26 & $\begin{array}{c}\text { Video motivacional NEPSO. } \\
\text { Discusión. }\end{array}$ \\
\hline \multirow[t]{2}{*}{$\begin{array}{l}\text { Definición y desarrollo del } \\
\text { tema }\end{array}$} & \multirow[t]{2}{*}{ Mayo } & 3 & $\begin{array}{c}\text { Dinámica “Yo doy una mano con..." resumen cualidades personales } \\
\text { y proposición de los temas de interés. } \\
\text { División por temáticas. } \\
\text { Discusión socializada sobre los temas de interés escogidos. }\end{array}$ \\
\hline & & 11 & $\begin{array}{l}\text { Revisión de textos de estudio y material aportado por las tesistas. } \\
\text { Selección de tópico a investigar: “Conducta adolescente”. } \\
\text { Votación para la determinación del tema: “Sexualidad y embarazo adolescente”. }\end{array}$ \\
\hline \multirow{4}{*}{$\begin{array}{l}\text { Definición de la población y } \\
\text { de la muestra }\end{array}$} & & 24 & Proceso de investigación: opiniones personales, lectura de libros. \\
\hline & & 31 & $\begin{array}{l}\text { Formulación de hipótesis. ¿Por que a pesar de haber tanta información las jóvenes } \\
\text { adolescentes se embarazan? (por soledad, porque aún falta información, poca } \\
\text { relación familiar, mala atención del servicio de salud, poco interés del gobierno por } \\
\text { fomentar la educación sexual). }\end{array}$ \\
\hline & \multirow[t]{5}{*}{ Junio } & 1 & $\begin{array}{l}\text { Visita de Ana Lima, Directora del Instituto Paulo Montenegro, programa NEPSO } \\
\text { IBOPE / IPM / AC̣ÃO EDUCATIVA. } \\
\text { Definición de la población y de la muestra. }\end{array}$ \\
\hline & & 7 & $\begin{array}{l}\text { Intervenciones en la clase de ciencias sociales, profesora Gloria Marivil: } \\
\text { Cosmovisión Mapuche y otras culturas. }\end{array}$ \\
\hline \multirow{11}{*}{$\begin{array}{l}\text { Elaboración de los cues- } \\
\text { tionarios }\end{array}$} & & 14 & Lectura y discusión de materiales aportados por las alumnas. Temas variados. \\
\hline & & 21 & Celebración Wetripantü (año nuevo mapuche) \\
\hline & & 28 & Clase magistral embarazo y sistema endocrino. \\
\hline & \multirow[t]{4}{*}{ Julio } & 5 & Materia: control hormonal, hipotálamo, hipófisis. Aparato reproductor. \\
\hline & & 12 & Materia: ciclo menstrual y ovulación. \\
\hline & & 19 & Receso invernal. \\
\hline & & 26 & Receso invernal. \\
\hline & \multirow[t]{4}{*}{ Agosto } & 9 & Afectividad y autoestima. El sentido de lo humano. Humberto Maturana. Discusión. \\
\hline & & 16 & $\begin{array}{l}\text { Determinación final del tema y elección de sub-temas. } \\
\text { Elaboración de preguntas de encuesta. “qqué queremos saber?”. }\end{array}$ \\
\hline & & 23 & $\begin{array}{c}\text { Elaboración de preguntas para el cuestionario. El curso se divide en grupos } \\
\text { pequeños con los sub-temas a elección. }\end{array}$ \\
\hline & & 30 & $\begin{array}{c}\text { Alumnos analizan preguntas en su grupo, para luego exponerlas al curso, } \\
\text { procediendo a la evaluación y corrección en conjunto. } \\
\text { Construcción del cuestionario final. }\end{array}$ \\
\hline \multirow[t]{4}{*}{ Trabajo de campo } & \multirow[t]{4}{*}{ Septiembre } & 6 & $\begin{array}{l}\text { Aplicación del pre-test en el mismo establecimiento, a los compañeros de primer } \\
\text { ciclo de enseñanza media. } \\
\text { Se identificaron los errores del cuestionario, modificando y eliminando preguntas; } \\
\text { se verificó el tiempo estimativo de la aplicación, los encuestados evaluaron la } \\
\text { experiencia, luego los encuestadores compartieron su apreciación. } \\
\text { Se realizan gestiones con los directores de establecimientos educacionales de } \\
\text { Nueva Imperial (Príncipe de Gales; Luís González Vásquez). }\end{array}$ \\
\hline & & 20 & Receso fiestas patrias. \\
\hline & & 27 & Últimas correcciones, encuesta. \\
\hline & & 1.4 & Aplicación encuestas en los liceos y en la plaza de armas. \\
\hline \multirow[t]{2}{*}{$\begin{array}{l}\text { Tabulación y procesamiento } \\
\text { de las informaciones. }\end{array}$} & \multirow[t]{2}{*}{ Octubre } & $8-24$ & $\begin{array}{c}\text { Tabulación de datos, construcción de gráficos. Profesores involucrados: Irene } \\
\text { Huentemilla, Ciencias Naturales, y Daniel Toro, de Matemáticas. }\end{array}$ \\
\hline & & 25 & Visita de Secretario Regional Ministerial de Salud (S), Claudio Obando. \\
\hline \multirow{3}{*}{$\begin{array}{l}\text { Análisis e interpretación de } \\
\text { los resultados }\end{array}$} & \multirow[t]{3}{*}{ Noviembre } & 1 & Feriado legal \\
\hline & & 8 & Interpretación y proceso de análisis de los datos tabulados. \\
\hline & & 15 & Exposición de resultados al curso. \\
\hline $\begin{array}{l}\text { Presentación de los datos } \\
\text { con gráficos } \\
\text { Sistematización y divul- } \\
\text { gación de los resultados }\end{array}$ & Diciembre & 6 & $\begin{array}{l}\text { Tres alumnos representantes del CEIA presentaron el trabajo realizado en la } \\
\text { primera Jornada de Intercambio de Experiencias entre los establecimientos } \\
\text { educacionales participantes del Proyecto NEPSO Polo CHILE, } \\
\text { Universidad de la Frontera, Temuco, Chile. }\end{array}$ \\
\hline
\end{tabular}


El proyecto aplicado siguió los pasos metodológicos definidos por el manual y, en general, por los diseños que se refieren a la encuesta de opinión (MONTENEGRO, FABIO \& MASAGAO, VERA, 2002). Se utiliza la metodología de acuerdo a lo presupuestado en el manual, sin embargo, en las actas de las reuniones y sistematizaciones, se señala que, a pesar de respetarla, se reconocen varias deficiencias de los profesores en términos de metodología de investigación, particularmente en la determinación de la pregunta de investigación, la selección de la muestra y el análisis de la información recogida. (WILLIAMSON, 2008)

\subsection{Resultados}

El estudio recogió observaciones - desde una pauta técnica con indicadores - a los participantes del curso al inicio y al final del desarrollo del proyecto. A continuación se presenta un cuadro resumen con cada problema-indicador diagnosticado al inicio del proceso contrastado con los cambios observados al término de la etapa de trabajo de campo, esta síntesis es una recopilación de la información obtenida (que no es cuantificable) por los distintos instrumentos de evaluación ya señalados. El énfasis está en determinar cambios producidos y no en lo que no se logró. 


\section{CUADRO SITUACIÓN INICIAL Y FINAL DEL PROYECTO}

SITUACIÓN INICIAL

Bajo nivel de comprensión lectora.

\section{LENGUAJE}

$\begin{gathered}\text { Dificultad para emitir discursos orales de carácter } \\ \text { argumentativo y expositivo. }\end{gathered}$
Problemas para la producción de textos escritos.
Dificultades para producir textos con coherencia
y cohesión.

MATEMÁTICAS

Problemas para aplicar operatoria básica frente a la
resolución y comprensión de problemas de la
vida diaria y entorno.
vida diaria y entorno.

\section{SITUACIÓN FINAL}

Presentan mejoras significativas en este ámbito. Comprenden textos, tanto de carácter literario como de carácter científico, con mayor facilidad que al comienzo del proyecto, cuando era muy difícil de enfrentar la comprensión lectora. Enfrentan al público en emisiones de discursos expositivos y foros argumentativos, exponiendo y defendiendo el tema investigado. Son capaces de producir preguntas de carácter científico para la encuesta de opinión.

Son capaces de valorar y evaluar las preguntas que son más pertinentes para los objetivos planteados para la investigación. Formulan objetivos e hipótesis de trabajos claros y coherentes con los temas de la investigación. Exponen y defienden su investigación ante audiencia. Los alumnos y alumnas son capaces de realizar cálculos matemáticos con facilidad, sacar porcentajes y relaciones matemáticas aplicadas.

Los alumnos y alumnas, al investigar el tema del embarazo adolescente, fueron expuestos e inmersos en una red de conocimientos que incluyeron conocimientos científicos (como el funcionamiento de los ciclos y diferentes aparatos del sistema reproductor) tanto como las relaciones que existen entre ese tipo de conocimiento y las prácticas humanas.

\begin{tabular}{ccc}
\hline TECNOLOGía & $\begin{array}{c}\text { Presentan escaso dominio de computadora. } \\
\text { Bajo o nulo manejo de programas de edición de texto } \\
\text { (Microsoft Word y Microsoft Power Point) y hojas de cálcu- } \\
\text { lo (Microsoft Excel) y navegadores de Internet. }\end{array}$ & $\begin{array}{c}\text { Los alumnos y alumnas son capaces de utilizar programas } \\
\text { de edición de textos para escribir y dar formato a la encues- } \\
\text { ta de opinión. Además de eso, emplean el Microsoft Excel } \\
\text { para procesar, tabular los datos y construir gráficos. } \\
\text { Utilizan internet para la búsqueda de información } \\
\text { pertinente al tema de su investigación. }\end{array}$ \\
\hline MOTIVACIONAL & $\begin{array}{c}\text { Presentan poca motivación, en especial la mayoría de los } \\
\text { alumnos que no conocen el proyecto. }\end{array}$ & $\begin{array}{c}\text { Al finalizar el proyecto los alumnos se sienten satisfechos, } \\
\text { orgullosos del conocimiento generado, dimensionan todo } \\
\text { el trabajo que hubo detrás de cada etapa. Hay un }\end{array}$ \\
& $\begin{array}{c}\text { proceso metacognitivo en ellos, ya que identifican la forma } \\
\text { como aprenden mejor, la motivación que les produce } \\
\text { investigar un tema que parte de sus intereses. }\end{array}$
\end{tabular}

Lo que se verifica desde esta observación es que en los sectores considerados y, particularmente en el de lenguaje, hay aprendizajes concebidos como cambios, en los indicadores que se describen en ambas columnas. En ello la metodología propuesta y desarrollada impulsa aprendizajes cognitivos, instrumentales, sociales y valóricos.

\section{Análisis}

Incorporar nuevas didácticas o nuevas formas de enseñar y/o aprender en el aula es una difícil tarea, no tan sólo para los profesores, 
quienes deben diseñar o planificar estas actividades de manera que contribuyan al aprendizaje de los alumnos, sino también para los educandos. Estos últimos, bajo la concepción constructivista de la educación, deben aprehender comprensivamente el conocimiento y, luego de un arduo proceso cognoscitivo, apoderarse del mismo para lograr decir: yo sé, yo aprendí, yo construí este conocimiento, lo construimos juntos con mis profesores y mis compañeros, investigando el medio a partir de lo que sabíamos de él convertido en una pregunta que nos impulsó a saber más.

Esta concepción, el constructivismo, se define como un enfoque psicopedagógico - no exento de crítica política (TADEU DA SILVA, 1998) - en el que el sujeto construye el conocimiento mediante la interacción que sostiene con el medio social y físico. Para Vygotsky este proceso se logra gracias al uso de la palabra, pues es el lenguaje el que permite la elaboración del conocimiento, el desarrollo mismo del pensamiento, dando origen al pensamiento consciente y a la elaboración de conceptos, lo que contribuye a que el aprendizaje se adelante al desarrollo y, más aún, lo permita. Otra dimensión de su teoría es la conceptualización del aprendizaje como un proceso social en que la ayuda del otro es el que permite alcanzar niveles superiores de pensamiento: lo que denomina zona de desarrollo próximo (SARRAMONA, 2008). Vygotsky, aunque refiriéndose a los niños, su objeto de estudio, plantea que el desarrollo "es un proceso dialéctico complejo caracterizado por la periodicidad, la irregularidad en el desarrollo de las distintas funciones, la metamorfosis o transformación cualitativa de una forma en otra, la interrelación de factores internos y externos, y los procesos adaptativos que superan y vencen los obstáculos con los que se cruza." (VYGOTSKY, 2009. p. 116) En su concepción, el desarrollo es comprendido como un proceso complejo no-lineal constituido por dinámicas dialécticas de cambio y ruptura, de evolución y revolución. El autor plantea que:

para la mente ingenua, revolución y evolución parecen incompatibles y el desarrollo histórico continúa únicamente mientras sigue una línea recta. Cuando se producen trastornos, o cuando se rompe el tejido histórico, la mente ingenua ve tan sólo catástrofes, vacíos y discontinuidad (...) Por el contrario, el pensamiento científico considera la revolución y la evolución como dos formas de desarrollo mutuamente relacionadas, de las que una presupone la otra (...) la mente científica los conceptúa como un momento en la línea general del desarrollo. (VYGOTSKY, 2009, p. 116, 117). 
En la misma línea, Paulo Freire afirma que la "conciencia transitiva es, $(. .$.$) en un primer estado, preponderantemente ingenua (. .$.$) se$ caracteriza, entre otros aspectos, por la simplicidad en la interpretación de los problemas", por otra parte, la

transitividad crítica (...) a que llegaríamos con una educación dialogal y activa, orientada hacia la responsabilidad social y política, se caracteriza por la profundidad en la interpretación de los problemas (...) por la receptividad de lo nuevo, no sólo por nuevo y por la no-negación de lo viejo, sólo por viejo, sino por la aceptación de ambos, en cuanto a su validez (FREIRE, 1971, p. 67-68).

"Es este choque entre un ayer diluyéndose, pero queriendo permanecer, y un mañana por consustanciarse, lo que caracteriza el tránsito de un tiempo anunciador" (FREIRE, 1971, p.46). Desde una perspectiva biosistémica, estar vivos y construir conocimiento implica una interrelación íntima entre la vida, el diálogo y la construcción de conocimiento, entre vida planetaria, transformaciones evolutivas y la forma en que nos situamos en los cambios: hacer investigación entonces es estar conectado y relacionado (NOGUEIRA, 2009).

Estas preocupaciones están en la base de lo que se aprende en el proyecto en cuanto una tarea social de desarrollo cognitivo, instrumental, valórico y social a través de la investigación. Es cierto que el aprendizaje en los adultos tiene ciertas características particulares (UNDURRAGA I., 2007), pero también que las teorías psicopedagógicas intentan explicar proyectivamente sus definiciones respecto de la infancia hacia el mundo adulto y juvenil. Por otra parte, si bien no se puede distinguir radicalmente entre educación "preadulta" y educación "de adultos" en una perspectiva de educación permanente y de estrategias de enseñanza-aprendizaje,

sin duda los adultos tienen perfiles específicos que justifican una atención diferenciada. Tales perfiles se concretan básicamente en las características de madurez y vinculación al medio social, que conducen hacia la autonomía (...) y como común denominador la experiencia que obliga a plantear todo nuevo aprendizaje en función de las vivencias y aprendizajes ya adquiridos (SARRAMONA, 2008, p. 121).

La realidad educacional chilena, con respecto al constructivismo, es ambivalente y más bien discursiva. Según se ha observado en los diver- 
sos eventos de perfeccionamiento en educación de adultos que desarrolla la universidad, dentro del pensamiento y discurso colectivo de los profesores, se cree que este nuevo paradigma es complejo, difícil de llevar a cabo en su totalidad, que en la praxis sólo se pueden trabajar aspectos aislados, que el constructivismo en el aula es casi imposible, es una utopía pedagógica más.

Las metodologías de proyectos de aula a través de la investigación con el instrumento encuesta de opinión, como la que propone el proyecto NEPSO, ofrece una opción innovadora, que permite "aterrizar" el constructivismo: hacer de la teoría una práctica y llevarla a las aulas. La encuesta de opinión como herramienta didáctica permite integrar líneas de acción pertinentes a este nuevo esquema, por lo tanto, se crea el espacio para que los alumnos puedan aportar sus dudas, sus preguntas, expresar sus discrepancias, mostrar sus conocimientos, plantear sus propuestas, preguntar en un clima contenedor y criticar, seguros de que sus aportes serán respetados y tenidos en cuenta. Además, permite fortalecer la interdisciplinaridad, contextualiza los contenidos de las diferentes asignaturas, integra a la comunidad en el proceso de enseñanza-aprendizaje, es por lo tanto, productora de conocimiento, logrando así el aprendizaje significativo de los alumnos.

El NEPSO presentó, para los jóvenes y adultos del $2^{\circ}$ ciclo de enseñanza media, una oportunidad de generación de conocimientos y de crecimiento personal profundo. Las áreas del conocimiento involucradas en el desarrollo de la metodología permitieron que alumnos y alumnas lograsen un avance oportuno para satisfacer necesidades de los mismos como jóvenes y adultos insertos en el ámbito laboral y socialmente activos.

La importancia del lenguaje en las prácticas educativas y sociales es innegable. Generalmente se relaciona el nivel de comprensión de lectura con el rendimiento académico, es por ello que se observa en este proyecto una excelente oportunidad para acrecentar los conocimientos y las experiencias en este ámbito: aprender a leer comprendiendo lo que se lee es una tarea que aparentemente se vislumbra fácil, sin embargo, la mayoría de los estudiantes chilenos no entienden lo que leen. Cuando se habla de entender, se está diciendo que los alumnos y alumnas se adueñen de la esencia de un texto, que cuando "lean" una imagen, sean capaces de descifrar el mundo que los rodea, recordando que la lectura del mundo precede a la lectura de la palabra (FREIRE, 1988). Por ello la búsqueda del 
conocimiento se encuentra en el diálogo dialéctico entre el contenido cultural del mundo que está en la memoria del "yo" y el mundo del que formo parte y está abierto a ser re-construido por el pensamiento en cada acto humano, sobre todo en los sistemáticos orientados a conocer y descubrir, desde el asombro, nuevos contenidos del mundo, convertidos en culturales, con una metodología que lo facilita y convierte en saber organizado y comunicable. Los estudiantes que participaron del proyecto NEPSO fueron capacitados en/con/por cada etapa que experimentaron para el desarrollo de la metodología, teniendo importantes avances en el uso del lenguaje. Esto les trajo, claramente, varios beneficios, no tan sólo para su etapa académica, sino que también para su desarrollo personal y social (y en esta dimensión su carácter ciudadano y trabajador), ya que aprenden a expresarse con mayor claridad y libertad, utilizando distintos medios: las expresiones oral, gestual, artística y audiovisual. En el establecimiento, en las calles y barrios, desarrollan una comunicación fluida y propositiva, expresando en claras imágenes a un ser humano capaz de vivir en sociedad.

\section{En conclusión}

Practicando esta didáctica, los alumnos y los profesores viven procesos democráticos significativos dentro de la producción del conocimiento, esto es, porque al consultar la opinión del otro practican la tolerancia y, básicamente, las personas que integran estos procesos democráticos tienen la posibilidad de influir abiertamente y de manera natural sobre el proceso de toma de decisiones del currículo haciéndolo a su vez participativo.

Los integrantes del proyecto manifestaron que se sintieron autores y generadores de su propio aprendizaje, esto se observó en todas las etapas de la implementación de la investigación, pero se incrementó principalmente al finalizar el proyecto, cuando comienzan a ver resultados tangibles de la investigación. En los alumnos y alumnas se produce un proceso metacognitivo que les permite dimensionar el conocimiento generado y se dan cuenta de que así aprenden mejor, que el método de investigación de encuestas de opinión les hace más interesante el aprender. La motivación es mayor para los alumnos en este proceso, según ellos indi- 
can, en la medida que el tema del trabajo parte de sus propios intereses y motivaciones.

La diversidad intergeneracional, étnica y de género encuentra en la investigación con encuesta de opinión - por su carácter metodológico participativo - una estrategia de formación en torno a un eje temático de interés compartido y que a todos - a unos más y otros menos - les moviliza en el proceso de aprendizaje. Este factor no es un detalle, sin duda que en contextos de alta diversidad, al menos en estas tres dimensiones: generacional, étnica y de género, los procesos de aprender se complejizan en sus relaciones sociales y sentidos, pero sobre todo y desde la perspectiva de lo que aquí se discute, una de las principales dificultades hoy es la disposición de un instrumental que permita el trabajo pedagógico de la diversidad para los profesores y profesoras. No hay apoyo metodológico en contextos de multiculturalidad y diversidad para docentes formados en un tiempo en que lo que se valoraba era la homogeneidad, el etnocentrismo, el currículo formal oficial, la igualdad republicana otorgada por el estadonación. No sirven recomendaciones de supervisores o académicos del tipo "profesor: adecúe...adapte". Sin duda que es un aporte a los docentes una metodología que permita que estudiantes muy diversos puedan alcanzar consensos democráticos sobre temas significativos y que justamente desde sus diferentes memorias personales y colectivas puedan construir preguntas comunes de interés general y particular, reunir información y darles respuestas desde la reflexión y el análisis. Se respeta, aprovecha y potencia pedagógicamente el diálogo productor de conocimiento entre la cultura educacional oficial del currículo y la cultura que otorga identidad al estudiante, contribuyendo a resolver la relación entre la identidad cultural, el diálogo entre esas identidades y el itinerario educativo. De esa manera, se supera una noción asimilacionista de adquisición de paquetes de conocimiento - evaluados según asumen ese paquete - por otra centrada en el desarrollo de la capacidad de pensar con autonomía (GADOTTI, 1996). Respecto de los profesores, siempre se dijo que ellos "deben respetar la cultura del alumno - su currícula oculta -, pero pocos instrumentos fueron señalados para auxiliar a los maestros en esa tarea (...) generalmente nuestra enseñanza se dirige a un alumno medio que es una abstracción de un alumno real". Una educación que reconoce y practica la diversidad le habla a un estudiante concreto, "valoriza la perspectiva del alumno, abriendo el sistema escolar, y construyendo una currícula 
más próxima a su realidad cultural" (GADOTTI, 1996, p. 192-193). Es lo que se observó en el proyecto metodológico de investigación y por ello el CEIA en su proyecto educativo anual, "El currículo y prácticas pedagógicas", continúa trabajando con la propuesta metodológica de investigación con encuesta de opinión NEPSO.

El conocimiento generado por los alumnos estimula la transformación social local o comunitaria, ya que, en los proyectos de investigación, surgen temas de cuño social como los religiosos, profesionales, sexuales, familiares, entre otros. La integración de la comunidad, el intercambio que se produce entre escuela y comunidad hace del proyecto un espacio para la reflexión permanente, que los alumnos encuesten en su comuna y luego presenten los resultados de su investigación, se traduce en que la escuela salga a la comunidad y rompa con una barrera existente. Es dentro de este ámbito donde se observaron también el importante crecimiento en los ámbitos motivacionales de los estudiantes participantes del Proyecto NEPSO, lo que es un importante aliciente para futuras prácticas de la metodología.

Hay diversas concepciones educacionales que asumen una reestructuración del currículo hacia la integración de contenidos culturales disciplinarios y de la escuela en su relación con las comunidades locales, concebidas éstas como territorios de aprendizajes interculturales (WILLIAMSON, 2005). Currículo interdisciplinario, currículo globalizado, currículo transversal, currículo coherente, centros de interés, abordaje de métodos de proyectos y solución de problemas, unidades didácticas integradas, etc en definitiva se engloban en la noción de currículo integrado asociado a la idea de aprendizaje significativo y contextualización curricular. Son tres los tipos de argumentación que lo justifican:

1) argumentaciones acerca de la necesidad de una mayor interrelación entre las diferentes disciplinas o materias; 2) atención a aquellas peculiaridades cognitivas y afectivas de los niños que influyen en sus procesos de aprendizaje y 3) la necesidad de que se lleve en consideración la comunidad en la cual la escuela está integrada; la necesidad de estar abierto a otras comunidades y lugares del mundo (...) (JURJO, 1996, p. 59). ${ }^{11}$

La integración curricular llevada a cabo da cuenta que la praxis de los asuntos teóricos es posible. En cuanto al carácter interdisciplinario que proporciona el NEPSO, se puede mencionar que uno de los puntos 
más significativos es el notorio compromiso y cooperación docente que logra fortalecerse con cada paso que el proyecto va desarrollando. La metodología facilita y permite que los docentes. Este quehacer amplía indudablemente la apreciación de los alumnos hacia la continuidad de los contenidos tratados en cada asignatura y hace tangibles los usos de cada disciplina, trasformando estos saberes algo útil para satisfacer sus interrogantes. Además, fortalece el trabajo en equipo de los profesores, reduce la fragmentación del currículum, promueve la profundidad en los procesos de enseñanza-aprendizaje, involucra al estudiantado en un aprendizaje activo y estimula los niveles más complejos del pensamiento. Sin duda que hay también aspectos que se deben mejorar, particularmente algunos metodológicos, como la definición del tema y problema, la definición del universo y determinación de la muestra, el análisis de resultados. Ello lleva a la necesidad de que los profesores se perfeccionen y en la formación inicial se integren metodologías participativas de investigación. Pero todo lo anterior confluye en el bien más preciado de todo educador: el aprendizaje significativo de sus alumnos y alumnas, que ven concretados sus anhelos en una investigación que comienza desde sus propios intereses. 


\section{REFERÊNCIAS}

BARRÍA CORTEZ, Pamela Josefina. NEPSO, una evaluación de la experiencia pedagógica. Temuco: Universidad de La Frontera. Tesis presentada para obtener el Grado de Magíster en Educación Mención Evaluación Educacional, 2008.

DURÁN MONDACA, Natalia Elisabet; TORRES BRAVO, Issa Johanna. Innovación Educativa. Proyecto NEPSO, Nuestra Escuela Pregunta Su Opinión. Evaluación de la Experiencia CEIA Lefxarv. Nueva Imperial, Región de La Araucanía, [s.n.], 2008.

FREIRE, Paulo. La Educación como Práctica de la Libertad. 4. ed. Montevideo: Tierra Nueva. Cuarta Edición, 1971.

FREIRE, Paulo. A Importância do Ato de Ler: em três artigos que se completam. 22 ed. São Paulo: Cortez, 1988.

GADOTTI, Moacir. Pedagogía de la Praxis. Buenos Aires: Miño y Dávila Editores S.A, 1996.

INSTITUTO PAULO MONTENEGRO. Almanaque NEPSO. São Paulo: Instituto Paulo Montenegro, Ação Educativa, 2007.

MONTENEGRO, FABIO \& MASAGÃO, VERA (Ed.). Nossa escola pesquisa sua opinião: manual do professor. 2. ed. São Paulo: Global, 2002.

NOGUEIRA, Adriano. Filosofia, Ciência, Complexidade: questões para a educação. Porto Alegre: EDIPUCRS, 2009.

SARRAMONA, Jaume. Teoría de la Educación: reflexión y normativa pedagógica. Barcelona: Editorial Ariel, S. A, 2008.

TADEU DA SILVA, Tomaz (Org.). Liberdades reguladas: A pedagogia construtivista e outras formas de governo do eu. Petrópolis, RJ: Vozes, 1998.

TORRES SANTOMÉ, Jurjo. A instituição escolar e a compreensão da realidade: o currículo integrado. In: SILVA, Luiz Eron da. Reestruturação Curricular: novos mapas culturais, novas perspectivas educacionais. Porto Alegre: Sulina, 1996. p. 58-74.

UNDURRAGA INFANTE, Consuelo. ¿Cómo aprenden los adultos? Una mirada psicoeducativa. 2. ed. Santiago: Ediciones Universidad Católica de Chile, 2007.

VYGOTSKI, Lev S. E. El Desarrollo de los procesos psicológicos superiores. 3. ed. de Biblioteca de Bolsillo. Barcelona: Crítica, 2009.

WILLIAMSON, Guillermo (Ed.). Proyecto NEPSO: Aprendizajes de una experiencia de investigación en aula 2005-2007. Temuco, São Paulo: Universidad de La Frontera, Instituto Paulo Montenegro, TimeIbope, 2008.

WILLIAMSON, Guillermo. Territorios de aprendizajes interculturales: transitando a una nueva praxis pedagógica. In: Revista Pensamiento Educativo. Pontificia Universidad Católica de Chile-Facultad de Educación, 2005. v. 37, p. 163-181.

WILLIAMSON, Guillermo. Investigación Acción Participativa Intercultural en Comunidades Educacionales y Locales. Universidad de La Frontera, Proyecto Kelluwün, Temuco, 2002. 


\section{NOTAS}

${ }^{1}$ En portugués el Proyecto se denomina Nossa Escola Pesquisa Sua Opinião. En castellano se reemplazó la palabra "pesquisa" (investiga) por "pregunta" para mantener la sigla NEPSO. 2 http://www.peoplemeter.cl/timeibope/index.asp

3 www.ibope.com.br

4 http://www.ipm.org.br

5 http://www.acaoeducativa.org.br/portal

6 La comunidad académica y pedagógica NEPSO la integran Argentina (Universidad Nacional de Quilmes), Colombia (Fundación Antonio Restrepo Barco), México (PROED - Proeducación e IBOPE AGB México), Portugal (Instituto de Ciencias Sociales de la Universidad de Lisboa), Brasil (Instituto Paulo Montenegro del Instituto Brasileño de Opinión Pública y Estadística-IBOPE, ONG Ação Educativa, Proyecto de Enseñanza Fundamental de Jóvenes y Adultos/PROEF - Universidad Federal de Minas GeraisUFMG, Fundación de la Universidad Federal de Paraná para el Desarrollo de la Ciencia, de la Tecnología y de la Cultura-Funpar, Universidad Federal de Pernambuco, Programa de Postgrado en Educación de la Universidad Federal de Rio Grande do Sul-PPGE/UFRGS, Universidad de Caxias do Sul-UCS, Universidad Federal Rural de Pernambuco, Pontifícia Universidad Católica de Rio de Janeiro) y Chile (Universidad de La Frontera).

7 El apoyo financiero al proyecto resulta del aporte de la empresa TimeIbope, de los establecimientos y de la Universidad de La Frontera.

8 Es el nombre en Mapudungun o Mapucezugun (lengua del pueblo indígena mapuche) de su héroe Lautaro, cantado por Alonso de Ercilla y Zúniga en el poema épico La Araucana; significa "ave veloz". Agradecemos al CEIA Lefxaarv su genorosa colaboración para el desarollo del estudio que sirve de base a este artículo.

9 Esta información está basada en la Presentación que el CEIA Lefxarv hizo en el $2^{\circ}$ Encuentro Nacional del Proyecto NEPSO (Temuco, Octubre de 2009).

10 http://www.ipm.org.br/ipmb_pagina.php?mpg=3.07.00.00.00\&ver=por

11 Traducción: Guillermo Williamson.

12 Los docentes coordinadores de proyectos del 2008 fueron Irene Huentemilla (Directora y Biología) Gloria Marivil (Estudios Sociales) y Daniel Toro (Matemáticas).

13 Los docentes coordinadores del 2009 en el CEIA Lefxarv fueron Irene Huentemilla (Directora y Biología) y los docentes Rodrigo Manquean (Estudios Sociales y Oficios) e Issa Torres (Lenguaje). 14 http://www.ipm.org.br/ipmb_painel.php?mpg=3.04.01.00.00\&oq1=199\&oq2=NP\& oq3 $=$ NP\&oq $4=\mathrm{NP} \&$ oq $5=\mathrm{NP} \&$ ver $=$ por

Recebimento: $13 / 05 / 2010$ 